\title{
On small matrix subalgebras with a trivial centralizer *
}

\author{
Clément de Seguins Pazzis \\ Lycée Privé Sainte-Geneviève, 2, rue de l'École des Postes, 78029 Versailles Cedex, FRANCE.
}

\begin{abstract}
Given an integer $n \geq 3$, we investigate the minimal dimension of a subalgebra of $\mathrm{M}_{n}(\mathbb{K})$ with a trivial centralizer. It is shown that this dimension is 5 when $n$ is even and 4 when it is odd. In the latter case, we also determine all 4-dimensional subalgebras with a trivial centralizer.
\end{abstract}

AMS Classification : 15A45; 16S50.

Keywords : commutation, matrices, algebras, dimension, centralizer.

\section{Introduction}

Here, we set an integer $n \geq 2$ and a field $\mathbb{K}$. Using the French convention, we let $\mathbb{N}$ denote the set of non-negative integers and $\mathbb{N}^{*}$ the one of positive integers. We let $\mathrm{M}_{n}(\mathbb{K})$ denote the algebra of square matrices of order $n$ with entries in $\mathbb{K}$ and $T_{n}(\mathbb{K})$ its subalgebra of upper triangular matrices. We let $\mathrm{M}_{p, q}(\mathbb{K})$ denote the set of matrices with $p$ rows, $q$ columns and entries in $\mathbb{K}$.

All subalgebras of $\mathrm{M}_{n}(\mathbb{K})$ are required to contain the unit matrix $I_{n}$ : the subalgebra $\operatorname{Span}\left(I_{n}\right)$ will be called trivial.

For $(i, j) \in \llbracket 1, n \rrbracket^{2}$, we let $E_{i, j}$ denote the elementary matrix of $\mathrm{M}_{n}(\mathbb{K})$ with a zero entry in every position except for $(i, j)$ where the entry is 1 .

Given a subset $V$ of $\mathrm{M}_{n}(\mathbb{K})$, we let

$$
\mathcal{C}(V):=\left\{A \in \mathrm{M}_{n}(\mathbb{K}): \forall M \in V, A M=M A\right\}
$$

denote its centralizer, and we simply write $\mathcal{C}(A)=\mathcal{C}(\{A\})$ when $A$ is a matrix of $\mathrm{M}_{n}(\mathbb{K})$. Recall that $\mathcal{C}(V)$ is always a subalgebra of $\mathrm{M}_{n}(\mathbb{K})$ and that $\mathcal{C}(V)$ is

${ }^{*}$ E-mail address: dsp.prof@gmail.com 
also the centralizer of the subalgebra generated by $V$.

The Jordan matrix of order $n$ will be written $J_{n}=\left(\delta_{i+1, j}\right)_{1 \leq i, j \leq n}$, where $\delta_{a, b}$ equals 1 if $a=b$, and 0 otherwise.

In this paper, we will focus on subalgebras of $\mathrm{M}_{n}(\mathbb{K})$ with a small dimension and a trivial centralizer. The basic motivation for studying subalgebras with a trivial centralizer comes from the theory of representations of algebras. Let $\mathcal{A}$ be a subalgebra of $\mathrm{M}_{n}(\mathbb{K})$, which we identify with the algebra of linear endomorphisms of the vector space $\mathbb{K}^{n}$. This yields a structure of $\mathcal{A}$-module on $\mathbb{K}^{n}$ for which the endomorphisms naturally correspond to the matrices in the centralizer $\mathcal{C}(\mathcal{A})$. When $\mathbb{K}$ is algebraically closed and $\mathbb{K}^{n}$ is a simple $\mathcal{A}$-module (i.e. when it has no non-trivial submodule), then $\mathcal{C}(\mathcal{A})$ is trivial, however the converse may not hold. In the case $\mathcal{A}$ is generated by a finite subgroup of $\mathrm{GL}_{n}(\mathbb{K})$ and $\mathbb{K}$ has characteristic 0 , then the converse is classically true because $\mathcal{A}$ is then semi-simple (see the theory of linear representations of finite groups). In the general case of an arbitrary field and an arbitrary subalgebra of $\mathrm{M}_{n}(\mathbb{K})$, the condition $\mathcal{C}(\mathcal{A})=\operatorname{Span}\left(I_{n}\right)$ may thus be seen as an alternative notion of simplicity or semi-simplicity, which provides motivation enough for studying it systematically.

Non-trivial subalgebras of $\mathrm{M}_{n}(\mathbb{K})$ with a trivial centralizer are actually commonplace. A classical example is that of $T_{n}(\mathbb{K})$. Indeed, let $A \in \mathcal{C}\left(T_{n}(\mathbb{K})\right)$.

Then $A$ commutes with $E_{i, i}$ for every $i \in \llbracket 1, n \rrbracket$, which shows that $A$ is diagonal. The commutation of $A$ with $E_{1, i}$ for every $i \in \llbracket 2, n \rrbracket$ then shows that all diagonal entries of $A$ are equal.

It is somewhat harder to produce such subalgebras with a small dimension. Our main goal is to find the smallest dimension for such a subalgebra and to classify the subalgebras of minimal dimension.

Definition 1. We let $t_{n}(\mathbb{K})$ (or simply $t_{n}$ when the field is obvious) denote the smallest dimension of a subalgebra of $\mathrm{M}_{n}(\mathbb{K})$ with a trivial centralizer.

Notice first that a subalgebra of dimension 2 is always of the form $\mathbb{K}[A]$ for some matrix $A$ which is not a scalar multiple of $I_{n}$, so its centralizer contains $A$ and is therefore non-trivial. This essentially solves the case $n=2$.

Proposition 1. One has $t_{2}=3$. More precisely, $T_{2}(\mathbb{K})$ has a trivial centralizer and dimension 3.

We will assume $n \geq 3$ from now on. Our main results are stated below: 
Theorem 2. If $n \geq 3$ is even, then $t_{n}(\mathbb{K})=5$.

If $n \geq 3$ is odd, then $t_{n}(\mathbb{K})=4$.

Proposition 3. Let $p \in \mathbb{N} \backslash\{0,1\}$ and consider the linear subspace $\mathcal{F}_{2 p}$ of $M_{2 p}(\mathbb{K})$ generated by the matrices

$$
\left[\begin{array}{cc}
I_{p} & 0 \\
0 & 0
\end{array}\right],\left[\begin{array}{cc}
0 & 0 \\
0 & I_{p}
\end{array}\right],\left[\begin{array}{cc}
0 & I_{p} \\
0 & 0
\end{array}\right],\left[\begin{array}{cc}
0 & J_{p} \\
0 & 0
\end{array}\right] \text { and }\left[\begin{array}{cc}
0 & J_{p}^{t} \\
0 & 0
\end{array}\right]
$$

Then $\mathcal{F}_{2 p}$ is a 5-dimensional subalgebra of $M_{2 p}(\mathbb{K})$ with a trivial centralizer.

Proposition 4. Let $p \in \mathbb{N}^{*}$. Consider the matrices $C_{p}=\left[\begin{array}{ll}I_{p} & 0\end{array}\right]$ and $D_{p}=$ $\left[\begin{array}{ll}0 & I_{p}\end{array}\right]$ in $M_{p, p+1}(\mathbb{K})$, and define $\mathcal{H}_{2 p+1}$ as the linear subspace of $M_{2 p+1}(\mathbb{K})$ generated by the matrices

$$
\left[\begin{array}{cc}
I_{p} & 0 \\
0 & 0
\end{array}\right],\left[\begin{array}{cc}
0 & 0 \\
0 & I_{p+1}
\end{array}\right],\left[\begin{array}{cc}
0 & C_{p} \\
0 & 0
\end{array}\right] \text { and }\left[\begin{array}{cc}
0 & D_{p} \\
0 & 0
\end{array}\right] .
$$

Then $\mathcal{H}_{2 p+1}$ is a 4-dimensional subalgebra of $M_{2 p+1}(\mathbb{K})$ with a trivial centralizer.

Finally, we will prove that the latter example is essentially unique:

Proposition 5. Let $p \in \mathbb{N}^{*}$ and $\mathcal{A}$ be a subalgebra of $M_{2 p+1}(\mathbb{K})$ of dimension 4 with a trivial centralizer. Then $\mathcal{A}$ is conjugate to either $\mathcal{H}_{2 p+1}$ or its transposed subalgebra $\mathcal{H}_{2 p+1}^{t}$, i.e. there is a non-singular matrix $P \in G L_{2 p+1}(\mathbb{K})$ such that

$$
\mathcal{A}=P \mathcal{H}_{2 p+1} P^{-1} \quad \text { or } \quad \mathcal{A}=P \mathcal{H}_{2 p+1}^{t} P^{-1} .
$$

Remark 1 . It is an easy exercise to prove that $\mathcal{H}_{2 p+1}$ and $\mathcal{H}_{2 p+1}^{t}$ are not conjugate one to the other.

\section{Checking the examples}

We will start with a little lemma.

Lemma 6. Let $n \geq 2$ be an integer. Then $\operatorname{Span}\left(J_{n}, J_{n}^{t}\right)$ has a trivial centralizer.

Proof. Since $J_{n}$ is cyclic, its centralizer is $\mathbb{K}\left[J_{n}\right]$ (see [5] Theorem 5 p.23), and it thus contains only upper triangular matrices with equal diagonal entries. Similarly, every matrix of $\mathbb{K}\left[J_{n}^{t}\right]$ is lower triangular. It follows that every matrix in the centralizer of $\operatorname{Span}\left(J_{n}, J_{n}^{t}\right)$ must be scalar. 
The examples featured in Propositions 3 and 4 are based upon the same idea. Consider a decomposition $n=p+q$ and a linear subspace $V$ of $\mathrm{M}_{p, q}(\mathbb{K})$. It is then easily checked that

$$
\mathcal{H}:=\left\{\left[\begin{array}{cc}
a \cdot I_{p} & K \\
0 & b . I_{q}
\end{array}\right] \mid(a, b) \in \mathbb{K}^{2}, K \in V\right\}
$$

is always a subalgebra of $\mathrm{M}_{n}(\mathbb{K})$ with dimension $\operatorname{dim} V+2$. Straightforward computation also shows that the centralizer of the matrix $P=\left[\begin{array}{cc}I_{p} & 0 \\ 0 & 0\end{array}\right]$ is

$$
\mathcal{C}(P)=\left\{\left[\begin{array}{cc}
X & 0 \\
0 & Y
\end{array}\right] \mid(X, Y) \in \mathrm{M}_{p}(\mathbb{K}) \times \mathrm{M}_{q}(\mathbb{K})\right\} .
$$

Let $M=\left[\begin{array}{cc}X & 0 \\ 0 & Y\end{array}\right] \in \mathcal{C}(P)$. Then $M$ belongs to $\mathcal{C}(\mathcal{H})$ if and only if $\forall K \in$ $V, X K=K Y$. Of course, this last relation need only be tested on a basis of $V$.

From there, our claims may easily be proven.

\section{The example in Proposition 3.}

Here, $q=p$ and $V=\operatorname{Span}\left(I_{p}, J_{p}, J_{p}^{t}\right)$.

Let $(X, Y) \in \mathrm{M}_{p}(\mathbb{K})^{2}$ such that $X I_{p}=I_{p} Y, X J_{p}=J_{p} Y$ and $X J_{p}^{t}=J_{p}^{t} Y$. Then $Y=X$ and $X$ commutes with both $J_{p}$ and $J_{p}^{t}$. By Lemma $6, X$ is a scalar multiple of $I_{p}$ hence $\left[\begin{array}{cc}X & 0 \\ 0 & Y\end{array}\right]$ is a scalar multiple of $I_{n}$. This proves Proposition 3.

\section{The example in Proposition 4.}

Here $q=p+1$ and $V=\operatorname{Span}\left(C_{p}, D_{p}\right)$.

Let $(X, Y) \in \mathrm{M}_{p}(\mathbb{K}) \times \mathrm{M}_{p+1}(\mathbb{K})$ such that $\left[\begin{array}{cc}X & 0 \\ 0 & Y\end{array}\right] \in \mathcal{C}(\mathcal{H})$.

The identity $X C_{p}=C_{p} Y$ entails that $Y=\left[\begin{array}{cc}X & 0 \\ L & \alpha\end{array}\right]$ for some $(\alpha, L) \in \mathbb{K} \times$ $\mathrm{M}_{1, p}(\mathbb{K})$, whilst identity $X D_{p}=D_{p} Y$ shows that $Y=\left[\begin{array}{cc}\beta & L^{\prime} \\ 0 & X\end{array}\right]$ for some $\left(\beta, L^{\prime}\right) \in$ $\mathbb{K} \times \mathrm{M}_{1, p}(\mathbb{K})$. We thus have

$$
\left[\begin{array}{cc}
X & 0 \\
L & \alpha
\end{array}\right]=\left[\begin{array}{cc}
\beta & L^{\prime} \\
0 & X
\end{array}\right]
$$


Starting from the first column of $X$, an easy induction shows that $X$ is upper triangular with all diagonal entries equal to $\beta$. Also, starting from the last column of $X$, an easy induction shows that $L^{\prime}=0$ and $X$ is lower triangular.

This yields $X=\beta . I_{p}$ and $Y=\beta . I_{p+1}$ hence $\left[\begin{array}{cc}X & 0 \\ 0 & Y\end{array}\right]=\beta . I_{2 p+1}$, which proves Proposition 4.

\section{A lower bound for $t_{n}(\mathbb{K})$}

\subsection{Introduction}

Here, we will prove that $t_{n} \geq 4$ when $n$ is odd, and $t_{n} \geq 5$ when $n$ is even. In other words, we will prove that every subalgebra of $\mathrm{M}_{n}(\mathbb{K})$ has a non-trivial centralizer provided it has dimension $p \leq 3$ when $n$ is odd, and dimension $p \leq 4$ when $p$ is even. The proof is essentially laid out as follows:

- by extending the ground field, we reduce the study to the case of an algebraically closed field;

- in this case, we discard all unispectral subalgebras (i.e. subalgebras in which every operator has a sole eigenvalue);

- in the remaining cases, the considered subalgebra contains a non-trivial idempotent which we use to split the algebra $\mathcal{A}$ into several remarkable subspaces; we then use that splitting to find a non-scalar matrix in the centralizer of $\mathcal{A}$ when $\operatorname{dim} \mathcal{A}$ is small enough.

From now on, we set an integer $n \geq 3$ and a subalgebra $\mathcal{A}$ of $\mathrm{M}_{n}(\mathbb{K})$. The following elementary facts will be used repeatedly:

- for every $P \in \mathrm{GL}_{n}(\mathbb{K})$, the conjugate subalgebra $P \mathcal{A} P^{-1}$ has the same dimension as $\mathcal{A}$ and its centralizer $P \mathcal{C}(\mathcal{A}) P^{-1}$ is trivial if and only if $\mathcal{C}(\mathcal{A})$ is trivial.

- the transposed subalgebra $\mathcal{A}^{t}:=\left\{M^{t} \mid M \in \mathcal{A}\right\}$ has the same dimension as $\mathcal{A}$ and its centralizer $C(\mathcal{A})^{t}$ is trivial if and only if $C(\mathcal{A})$ is trivial.

\subsection{Reduction to the case of an algebraically closed field}

Let $\mathbb{L}$ be a field extension of $\mathbb{K}$. Recall that when $\mathcal{A}$ is a subalgebra of $\mathrm{M}_{n}(\mathbb{K})$ and we let $\mathcal{A}_{\mathbb{L}}$ denote the linear $\mathbb{L}$-subspace of $\mathrm{M}_{n}(\mathbb{L})$ generated by $\mathcal{A}$, then 
$\mathcal{A}_{\mathbb{L}}$ is a subalgebra of $\mathrm{M}_{n}(\mathbb{L})$. The natural isomorphism of $\mathbb{L}$-algebras $\mathrm{M}_{n}(\mathbb{L}) \simeq$ $\mathrm{M}_{n}(\mathbb{K}) \otimes_{\mathbb{K}} \mathbb{L}$ maps $\mathcal{A}_{\mathbb{L}}$ to $\mathcal{A} \otimes_{\mathbb{K}} \mathbb{L}$ hence $\operatorname{dim}_{\mathbb{L}} \mathcal{A}_{\mathbb{L}}=\operatorname{dim}_{\mathbb{K}} \mathcal{A}$. Also, the centralizer of $\mathcal{A} \otimes_{\mathbb{K}} \mathbb{L}$ in $\mathrm{M}_{n}(\mathbb{K}) \otimes_{\mathbb{K}} \mathbb{L}$ is clearly $\mathcal{C}(\mathcal{A}) \otimes_{\mathbb{K}} \mathbb{L}$, therefore $\mathcal{A}$ has a trivial centralizer in $\mathrm{M}_{n}(\mathbb{K})$ if and only if $\mathcal{A}_{\mathbb{L}}$ has a trivial centralizer in $\mathrm{M}_{n}(\mathbb{L})$. We deduce that

$$
t_{n}(\mathbb{K}) \geq t_{n}(\mathbb{L}) .
$$

Therefore, by Steinitz's theorem and the examples discussed earlier, it will suffice to prove theorem 2 when $\mathbb{K}$ is algebraically closed.

In the rest of this section, we assume $\mathbb{K}$ is algebraically closed.

\subsection{The case $\mathcal{A}$ is unispectral}

Definition 2. We call a matrix $A \in \mathrm{M}_{n}(\mathbb{K})$ unispectral when it has a sole eigenvalue.

A subalgebra of $\mathrm{M}_{n}(\mathbb{K})$ is called unispectral when all its elements are unispectral.

The standard example is the subalgebra of matrices of the form $\lambda \cdot I_{n}+T$ for some strictly upper triangular matrix $T$. Conversely, every unispectral subalgebra is conjugate to a subalgebra of the preceding one:

Proposition 7. Let $\mathcal{A}$ be a unispectral subalgebra of $M_{n}(\mathbb{K})$. Then there is a non-singular $P \in G L_{n}(\mathbb{K})$ such that $P \mathcal{A} P^{-1} \subset T_{n}(\mathbb{K})$.

Proof. We use Burnside's theorem (see [3] p.213) by induction on $n$. The case $n=1$ is trivial. Assume $n \geq 2$ and our claim holds for every non-negative integer $p<n$ and every unispectral subalgebra of $\mathrm{M}_{p}(\mathbb{K})$. Let $\mathcal{A}$ be a unispectral subalgebra of $\mathrm{M}_{n}(\mathbb{K})$. Clearly, $\mathcal{A} \subsetneq \mathrm{M}_{n}(\mathbb{K})$ hence Burnside's theorem shows there is a non-singular $P \in \mathrm{GL}_{n}(\mathbb{K})$ and an integer $p \in \llbracket 1, n-1 \rrbracket$ such that every $M \in \mathcal{A}$ splits as

$$
M=P^{-1}\left[\begin{array}{cc}
A(M) & * \\
0 & B(M)
\end{array}\right] P \quad \text { where } A(M) \in \mathrm{M}_{p}(\mathbb{K}) \text { and } B(M) \in \mathrm{M}_{n-p}(\mathbb{K}) .
$$

Then $\mathcal{A}_{1}:=\{A(M) \mid M \in \mathcal{A}\}$ (resp. $\mathcal{A}_{2}:=\{B(M) \mid M \in \mathcal{A}\}$ ) is a unispectral subalgebra of $\mathrm{M}_{p}(\mathbb{K})$ (resp. of $\mathrm{M}_{n-p}(\mathbb{K})$ ). Using the induction hypothesis, there are non-singular matrices $P_{1} \in \mathrm{GL}_{p}(\mathbb{K})$ and $P_{2} \in \mathrm{GL}_{n-p}(\mathbb{K})$ such that $P_{1} \mathcal{A}_{1} P_{1}^{-1} \subset T_{p}(\mathbb{K})$ and $P_{2} \mathcal{A}_{2} P_{2}^{-1} \subset T_{n-p}(\mathbb{K})$. Setting $Q:=\left[\begin{array}{cc}P_{1} & 0 \\ 0 & P_{2}\end{array}\right]$, we have $Q \in \mathrm{GL}_{n}(\mathbb{K})$ and $(Q P) \mathcal{A}(Q P)^{-1} \subset T_{n}(\mathbb{K})$. 
Corollary 8. Let $p \geq 2$ be an integer. Then every unispectral subalgebra of $M_{p}(\mathbb{K})$ has a non-trivial centralizer.

Proof. It suffices to prove the statement for any unispectral subalgebra $\mathcal{A}$ of $T_{p}(\mathbb{K})$. However any matrix $M$ of $\mathcal{A}$ must have identical diagonal entries and must be upper triangular: it easily follows that the elementary matrix $E_{1, n}$ lies in $\mathcal{C}(\mathcal{A})$.

In what follows, we will assume $\mathcal{A}$ is not unispectral.

\subsection{The basic splitting}

Let us choose a non-unispectral matrix $M \in \mathcal{A}$. Choose then a spectral projection $P$ associated to $M$ : hence $P \in \mathbb{K}[M] \subset \mathcal{A}$ (see [1] chapter 8,4 corollary 3 p.271) and we have therefore found a non-trivial idempotent in $\mathcal{A}$. By conjugating $\mathcal{A}$ with an appropriate non-singular matrix, we are reduced to the case $\mathcal{A}$ contains, for some $p \in \llbracket 1, n-1 \rrbracket$, the idempotent matrix

$$
P:=\left[\begin{array}{cc}
I_{p} & 0 \\
0 & 0
\end{array}\right] \in \mathrm{M}_{n}(\mathbb{K}) .
$$

Remark 2. The rest of the proof will only rely on this assumption and the condition on the dimension of $\mathcal{A}$. In particular, the reader will check that it does not use the fact that $\mathbb{K}$ is algebraically closed.

In what follows, we set $q:=n-p$. Notice then that $\mathcal{A}$ also contains $Q:=$ $I_{n}-P=\left[\begin{array}{cc}0 & 0 \\ 0 & I_{q}\end{array}\right]$. Since $\mathcal{A}$ is a subalgebra containing both $P$ and $Q$, one clearly has:

$$
\begin{aligned}
& P \mathcal{A} P=\left\{\left[\begin{array}{cc}
M & 0 \\
0 & 0
\end{array}\right] \mid M \in \mathrm{M}_{p}(\mathbb{K})\right\} \cap \mathcal{A} \quad, \quad P \mathcal{A} Q=\left\{\left[\begin{array}{cc}
0 & M \\
0 & 0
\end{array}\right] \mid M \in \mathrm{M}_{p, q}(\mathbb{K})\right\} \cap \mathcal{A}, \\
& Q \mathcal{A} P=\left\{\left[\begin{array}{cc}
0 & 0 \\
M & 0
\end{array}\right] \mid M \in \mathrm{M}_{q, p}(\mathbb{K})\right\} \cap \mathcal{A}, \quad \text { and } \quad Q \mathcal{A} Q=\left\{\left[\begin{array}{cc}
0 & 0 \\
0 & M
\end{array}\right] \mid M \in \mathrm{M}_{q}(\mathbb{K})\right\} \cap \mathcal{A} .
\end{aligned}
$$

Those four linear subspaces of $\mathcal{A}$ will respectively be denoted by $\mathcal{A}_{1,1}, \mathcal{A}_{1,2}, \mathcal{A}_{2,1}$ and $\mathcal{A}_{2,2}$. For every $M \in \mathrm{M}_{n}(\mathbb{K})$, write:

$$
M=I_{n} M I_{n}=(P+Q) M(P+Q)=P M P+P M Q+Q M P+Q M Q .
$$


It follows that

$$
\mathcal{A}=\mathcal{A}_{1,1} \oplus \mathcal{A}_{1,2} \oplus \mathcal{A}_{2,1} \oplus \mathcal{A}_{2,2}
$$

hence

$$
\operatorname{dim} \mathcal{A}=\operatorname{dim} \mathcal{A}_{1,1}+\operatorname{dim} \mathcal{A}_{1,2}+\operatorname{dim} \mathcal{A}_{2,1}+\operatorname{dim} \mathcal{A}_{2,2} .
$$

Notice also that $P \in \mathcal{A}_{1,1}$ and $Q \in \mathcal{A}_{2,2}$, so that

$$
\operatorname{dim} \mathcal{A}_{1,1} \geq 1 \quad \text { and } \quad \operatorname{dim} \mathcal{A}_{2,2} \geq 1
$$

In what follows, we will discuss various cases depending on the respective dimensions of the $\mathcal{A}_{i, j}$ 's. One case can readily be done away: if $\mathcal{A}_{1,2}=\{0\}$ and $\mathcal{A}_{2,1}=\{0\}$, then $P$ belongs to $\mathcal{C}(\mathcal{A}) \backslash \operatorname{Span}\left(I_{n}\right)$.

We will now assume $\mathcal{A}_{1,2} \neq\{0\}$ or $\mathcal{A}_{2,1} \neq\{0\}$.

\subsection{The case $\operatorname{dim} \mathcal{A}=3$}

It only remains to investigate the case one of $\mathcal{A}_{2,1}$ and $\mathcal{A}_{1,2}$ has dimension 1 and the other 0 . Transposition of $\mathcal{A}$ only leaves us with the case $\mathcal{A}_{2,1}=\{0\}$ and $\mathcal{A}_{1,2}$ is generated by some $C \in \mathrm{M}_{p, q}(\mathbb{K}) \backslash\{0\}$. Hence $\mathcal{A}_{1,1}=\operatorname{Span}\left(I_{p}\right)$ and $\mathcal{A}_{2,2}=\operatorname{Span}\left(I_{q}\right)$, so the considerations of Section 2 show that, in order to prove that $\mathcal{C}(\mathcal{A})$ is non-trivial, it will suffice to prove that, for some pair $(X, Y) \in \mathrm{M}_{p}(\mathbb{K}) \times \mathrm{M}_{q}(\mathbb{K})$, one has $X C=C Y$ and $\left[\begin{array}{cc}X & 0 \\ 0 & Y\end{array}\right]$ is not a scalar multiple of $I_{n}$. Consider then the linear map:

$$
f: \begin{cases}\mathrm{M}_{p}(\mathbb{K}) \times \mathrm{M}_{q}(\mathbb{K}) & \longrightarrow \mathrm{M}_{p, q}(\mathbb{K}) \\ (X, Y) & \longmapsto X C-C Y .\end{cases}
$$

By the rank theorem:

$\operatorname{dim} \operatorname{Ker} f \geq \operatorname{dim}\left(\mathrm{M}_{p}(\mathbb{K}) \times \mathrm{M}_{q}(\mathbb{K})\right)-\operatorname{dim} \mathrm{M}_{p, q}(\mathbb{K})=p^{2}+q^{2}-p q=(p-q)^{2}+p q \geq p q$.

Since $n \geq 3$ and $p \in \llbracket 1, n-1 \rrbracket$, one has $p q \geq 2$, which shows that Ker $f \neq$ $\operatorname{Span}\left(\left(I_{p}, I_{q}\right)\right)$. Therefore, $\mathcal{C}(\mathcal{A})$ is non-trivial. 


\subsection{The case $\operatorname{dim} \mathcal{A}=4$}

We now assume $\operatorname{dim} \mathcal{A}=4$ and set

$$
\nu(\mathcal{A})=\left(\operatorname{dim} \mathcal{A}_{1,1}, \operatorname{dim} \mathcal{A}_{1,2}, \operatorname{dim} \mathcal{A}_{2,1}, \operatorname{dim} \mathcal{A}_{2,2}\right) .
$$

Transposition and conjugation by a permutation matrix help us reduce the situation to only three cases:

- $\nu(\mathcal{A})=(2,1,0,1)$

- $\nu(\mathcal{A})=(1,1,1,1)$

- $\nu(\mathcal{A})=(1,2,0,1)$.

In the first two cases, we will show that $\mathcal{C}(\mathcal{A})$ is non-trivial, even when $n$ is odd. In the last case, we will show that $\mathcal{C}(\mathcal{A})$ is non-trivial when $n$ is even.

\subsubsection{The case $\nu(\mathcal{A})=(2,1,0,1)$.}

In this case, there is some $C \in \mathrm{M}_{p}(\mathbb{K}) \backslash \operatorname{Span}\left(I_{p}\right)$ and some $V \in \mathrm{M}_{p, q}(\mathbb{K}) \backslash\{0\}$ such that $\mathcal{A}$ is generated by the matrices

$$
\left[\begin{array}{cc}
I_{p} & 0 \\
0 & 0
\end{array}\right],\left[\begin{array}{cc}
0 & 0 \\
0 & I_{q}
\end{array}\right],\left[\begin{array}{cc}
C & 0 \\
0 & 0
\end{array}\right] \text { and }\left[\begin{array}{ll}
0 & V \\
0 & 0
\end{array}\right] .
$$

Since the product of the last two matrices belongs to $\mathcal{A}$, one must have $C V=\lambda V$ for some $\lambda \in \mathbb{K}$. Replacing $C$ with $C-\lambda . I_{p}$, we may assume $C V=0$, in which case the matrix $\left[\begin{array}{ll}C & 0 \\ 0 & 0\end{array}\right]$ clearly belongs to $\mathcal{C}(\mathcal{A}) \backslash \operatorname{Span}\left(I_{n}\right)$.

3.6.2 The case $\nu(\mathcal{A})=(1,1,1,1)$.

In this case, there are matrices $U \in \mathrm{M}_{q, p}(\mathbb{K}) \backslash\{0\}$ and $V \in \mathrm{M}_{p, q}(\mathbb{K}) \backslash\{0\}$ such that $\mathcal{A}$ is generated by the four matrices

$$
\left[\begin{array}{cc}
I_{p} & 0 \\
0 & 0
\end{array}\right],\left[\begin{array}{cc}
0 & 0 \\
0 & I_{q}
\end{array}\right],\left[\begin{array}{cc}
0 & 0 \\
U & 0
\end{array}\right] \text { and }\left[\begin{array}{ll}
0 & V \\
0 & 0
\end{array}\right]
$$

A matrix belongs to $\mathcal{C}(\mathcal{A})$ if and only if it has the form $\left[\begin{array}{cc}X & 0 \\ 0 & Y\end{array}\right]$, where $(X, Y) \in$ $\mathrm{M}_{p}(\mathbb{K}) \times \mathrm{M}_{q}(\mathbb{K})$ satisfies $U X=Y U$ and $X V=V Y$. 
One obvious element in this centralizer is $\left[\begin{array}{cc}V U & 0 \\ 0 & U V\end{array}\right]$. Assume now that $\mathcal{C}(\mathcal{A})$ is trivial. There would then exist some $\lambda \in \mathbb{K}$ such that $V U=\lambda . I_{p}$ and $U V=\lambda . I_{q}$. Two situations may arise:

- The case $\lambda \neq 0$. Replacing $V$ by $\frac{1}{\lambda} V$, we may assume $\lambda=1$. Then standard rank considerations show that $p=q$ and $V=U^{-1}$. Equality $X V=V Y$ thus implies $U X=Y U$, hence $\mathcal{A}$ has the same centralizer as the subalgebra generated by $P, Q$ and $\left[\begin{array}{ll}0 & V \\ 0 & 0\end{array}\right]$, which has been shown to be non-trivial in Section 3.5. There lies a contradiction.

- The case $\lambda=0$. Then $U V=0$ and $V U=0$. Since neither $U$ nor $V$ is zero, we deduce that $\operatorname{Ker} U$ and $\operatorname{Im} V$ are non-trivial subspaces of $\mathbb{K}^{p}$ and $\operatorname{Ker} V$ and $\operatorname{Im} U$ are non-trivial subspaces of $\mathbb{K}^{q}$. We can therefore construct rank 1 matrices $X \in \mathrm{M}_{p}(\mathbb{K})$ and $Y \in \mathrm{M}_{q}(\mathbb{K})$ such that $\operatorname{Im} X \subset \operatorname{Ker} U$, $\operatorname{Im} V \subset \operatorname{Ker} X, \operatorname{Im} Y \subset \operatorname{Ker} V$ and $\operatorname{Im} U \subset \operatorname{Ker} Y$. Hence $X V=V Y=0$ and $U X=Y U=0$, and it follows that the block diagonal matrix $\left[\begin{array}{cc}X & 0 \\ 0 & Y\end{array}\right]$ belongs to $\mathcal{C}(\mathcal{A}) \backslash \operatorname{Span}\left(I_{n}\right)$, another contradiction.

The previous reductio ad absurdum then proves that $\mathcal{C}(\mathcal{A})$ is non-trivial.

3.6.3 The case $\nu(\mathcal{A})=(1,2,0,1)$.

We actually lose no generality assuming $p \leq q$ (if not, we simply replace $\mathcal{A}$ with $\mathcal{A}^{t}$ and conjugate it by an appropriate permutation matrix). We then find linearly independent matrices $U$ and $V$ in $\mathrm{M}_{p, q}(\mathbb{K})$ such that $\mathcal{A}$ is generated by

$$
\left[\begin{array}{cc}
I_{p} & 0 \\
0 & 0
\end{array}\right],\left[\begin{array}{cc}
0 & 0 \\
0 & I_{q}
\end{array}\right],\left[\begin{array}{cc}
0 & U \\
0 & 0
\end{array}\right] \text { and }\left[\begin{array}{ll}
0 & V \\
0 & 0
\end{array}\right] \text {. }
$$

The considerations of Section 2 show that the kernel of

$$
g: \begin{cases}\mathrm{M}_{p}(\mathbb{K}) \times \mathrm{M}_{q}(\mathbb{K}) & \longrightarrow \mathrm{M}_{p, q}(\mathbb{K})^{2} \\ (X, Y) & \longmapsto(X U-U Y, X V-V Y)\end{cases}
$$

has a dimension greater than 1 if and only if $\mathcal{C}(\mathcal{A})$ is non-trivial.

Lemma 9. With the preceding notations, for to have $\operatorname{dim} \operatorname{Ker} g=1$, it is necessary that: 
- either $q=p$ and at least one of the matrices $U$ or $V$ has rank $p$;

- or $q=p+1$ and both matrices $U$ and $V$ have rank $p$.

In order to prove this lemma, we will start with another one:

Lemma 10. Let $W \in M_{p, q}(\mathbb{K})$ be such that $\operatorname{rk} W<p \leq q$. Then the linear map

$$
f: \begin{cases}M_{p}(\mathbb{K}) \times M_{q}(\mathbb{K}) & \longrightarrow M_{p, q}(\mathbb{K}) \\ (X, Y) & \longmapsto X W-W Y\end{cases}
$$

is not onto.

Proof. Notice that rk $f$ is unchanged by replacing $W$ with an equivalent matrix: for every $\left(P_{1}, Q_{1}\right) \in \mathrm{GL}_{p}(\mathbb{K}) \times \mathrm{GL}_{q}(\mathbb{K})$ and $(X, Y) \in \mathrm{M}_{p}(\mathbb{K}) \times \mathrm{M}_{q}(\mathbb{K})$, one can indeed write:

$$
X P_{1} W Q_{1}-P_{1} W Q_{1} Y=P_{1}\left[\left(P_{1}^{-1} X P_{1}\right) W-W\left(Q_{1} Y Q_{1}^{-1}\right)\right] Q_{1} .
$$

Letting $r:=\operatorname{rk} W$, we then lose no generality assuming that $W=\left[\begin{array}{cc}I_{r} & 0 \\ 0 & 0\end{array}\right]$. In this case however, every matrix of $\operatorname{Im} f$ has a zero entry in position $(p, q)$, hence $f$ is not onto.

Proof of Lemma 9. setting $f_{1}:(X, Y) \longmapsto X U-U Y$ and $f_{2}:(X, Y) \longmapsto X V-$ $V Y$, we find that $\mathrm{rk} g \leq \mathrm{rk} f_{1}+\mathrm{rk} f_{2}$ whilst Lemma 10 shows that $\mathrm{rk} f_{1}+\mathrm{rk} f_{2} \leq$ $2 \mathrm{M}_{p, q}(\mathbb{K})-m$ where $m$ is the number of matrices in $\{U, V\}$ which have rank lesser than $p$. We deduce that $\operatorname{rk} g \leq 2 p q-m$. The rank theorem then shows that

$$
\operatorname{dim} \operatorname{Ker} g \geq p^{2}+q^{2}-2 p q+m=(q-p)^{2}+m .
$$

If $q \geq p+2$, then $\operatorname{dim} \operatorname{Ker} g \geq 4$. If $q=p+1$ and $m \geq 1$, then $\operatorname{dim} \operatorname{Ker} g \geq 2$. If $q=p$ and $m=2$, then $\operatorname{dim} \operatorname{Ker} g \geq 2$. This proves the claimed results.

We may now complete the proof of Theorem 2. Assume $n$ is even. Then Lemma 9 shows $\mathcal{C}(\mathcal{A})$ is non-trivial unless $p=q=\frac{n}{2}$ and one of the matrices $U$ and $V$ is non-singular.

Assume then $p=q=\frac{n}{2}$ and $U$ is non-singular (for example). Conjugating $\mathcal{A}$ 
by $\left[\begin{array}{cc}I_{p} & 0 \\ 0 & U\end{array}\right]$, we are then reduced to the case $U=I_{p}$ : in this case $V \notin \operatorname{Span}\left(I_{p}\right)$ and the matrix $\left[\begin{array}{cc}V & 0 \\ 0 & V\end{array}\right]$ clearly belongs to $\mathcal{C}(\mathcal{A}) \backslash \operatorname{Span}\left(I_{n}\right)$.

Let us finish this section by summing up the results in the case $\operatorname{dim} \mathcal{A}=4$. Recall that we have assumed that $\mathbb{K}$ is algebraically closed or that $\mathcal{A}$ contains a non-trivial idempotent.

(i) If $n$ is even, then $\mathcal{C}(\mathcal{A})$ is non-trivial.

(ii) If $n$ is odd and $\mathcal{C}(\mathcal{A})$ is trivial, then, setting $p:=\frac{n-1}{2}$, there are linearly independent matrices $U$ and $V$ in $\mathrm{M}_{p, p+1}(\mathbb{K})$ with rank $p$ and a non-singular matrix $P \in \mathrm{GL}_{n}(\mathbb{K})$ such that either

$$
P \mathcal{A} P^{-1}=\left\{\left[\begin{array}{cc}
a . I_{p} & c . U+d . V \\
0 & b . I_{p+1}
\end{array}\right] \mid(a, b, c, d) \in \mathbb{K}^{4}\right\}
$$

or

$$
P \mathcal{A}^{t} P^{-1}=\left\{\left[\begin{array}{cc}
a . I_{p} & c . U+d . V \\
0 & b . I_{p+1}
\end{array}\right] \mid(a, b, c, d) \in \mathbb{K}^{4}\right\} .
$$

This of course completes the proof of Theorem 2 .

\section{On subalgebras of dimension 4 of $\mathrm{M}_{2 n+1}(\mathbb{K})$ with a trivial centralizer}

Here, we establish Proposition 5 by prolonging the proof of Section 3.6 in the case $\operatorname{dim} \mathcal{A}=4$. We must first return to the situation where $\mathbb{K}$ is an arbitrary field.

Lemma 11. Let $n \in \mathbb{N}^{*}$ and $\mathcal{A}$ be a 4-dimensional subalgebra of $M_{2 n+1}(\mathbb{K})$ with a trivial centralizer. Then $\mathcal{A}$ contains a rank $n$ idempotent.

Proof. Choose an algebraically closed extension $\mathbb{L}$ of $\mathbb{K}$. Then $\mathcal{A}_{\mathbb{L}}$ has a trivial centralizer in $\mathrm{M}_{2 n+1}(\mathbb{L})$. The proof in Sections 3.2, 3.3, 3.4 and 3.6 then shows that there is a 2-dimensional subspace $P \subset \mathrm{M}_{n, n+1}(\mathbb{K})$ such that $\mathcal{A}_{\mathbb{L}}$ is conjugate to either the subalgebra

$$
\mathcal{H}:=\left\{\left[\begin{array}{cc}
a . I_{n} & M \\
0 & b . I_{n+1}
\end{array}\right] \mid(a, b) \in \mathbb{L}^{2}, M \in P\right\}
$$


or its transpose $\mathcal{H}^{t}$. In any case, the set of unispectral elements in $\mathcal{A}_{\mathbb{L}}$ is a linear hyperplane of $\mathcal{A}_{\mathbb{L}}$ : this is the case indeed when $\mathcal{A}_{\mathbb{L}}=\mathcal{H}$ since this subset is then

$$
\left\{\left[\begin{array}{cc}
a \cdot I_{n} & M \\
0 & a \cdot I_{n+1}
\end{array}\right] \mid a \in \mathbb{L}, M \in P\right\} .
$$

Also, every non-unispectral element of $\mathcal{A}_{\mathbb{L}}$ is clearly diagonalisable with exactly two eigenvalues of respective orders $n$ and $n+1$.

Every basis of the $\mathbb{K}$-vector space $\mathcal{A}$ is also a basis of the $\mathbb{L}$-vector space $\mathcal{A}_{\mathbb{L}}$ and therefore must contain a matrix which is not unispectral in $\mathrm{M}_{n}(\mathbb{L})$. Let us choose such a matrix $M \in \mathcal{A}$, with eigenvalues $\lambda$ and $\mu$ of respective orders $n$ and $n+1$. Notice then that $\lambda$ and $\mu$ belong to $\mathbb{K}$. Indeed:

- the minimal polynomial of $M$ is $X^{2}-(\lambda+\mu) X+\lambda \mu$, so $\lambda+\mu \in \mathbb{K}$ (implicit here is the fact that the minimal polynomial of a matrix is unchanged by extending the field of scalars);

- also $\operatorname{tr}(M)=n(\lambda+\mu)+\mu$, which entails $\mu \in \mathbb{K}$ and therefore $\lambda \in \mathbb{K}$.

We deduce that $\frac{1}{\lambda-\mu}\left(M-\mu \cdot I_{2 n+1}\right)$ is a rank $n$ idempotent in $\mathcal{A}$.

Proposition 5 can now be proven. Since $\mathcal{A}$ contains an idempotent of rank $n$, the proof from Sections 3.4 and 3.6 shows that we can reduce the study to the situation where there is a 2-dimensional subspace $\mathcal{P} \subset \mathrm{M}_{n, n+1}(\mathbb{K})$ such that

$$
\mathcal{A}=\left\{\left[\begin{array}{cc}
a . I_{n} & M \\
0 & b . I_{n+1}
\end{array}\right] \mid(a, b) \in \mathbb{K}^{2}, M \in \mathcal{P}\right\}
$$

Let $U \in \mathcal{P} \backslash\{0\}$ and choose $V$ such that $(U, V)$ is a basis of $\mathcal{P}$. Then Lemma 11 shows that $U$ (and $V$ ) must have rank $n$. For every extension $\mathbb{L}$ of $\mathbb{K}$, the subalgebra $\mathcal{A}_{\mathbb{L}}$ has a trivial centralizer, which shows $\operatorname{rk} U=n$ for every $U \in$ $P_{\mathbb{L}} \backslash\{0\}$. We will then use the following result to see that $\mathcal{P}$ is equivalent to the 2-dimensional subspace $\operatorname{Span}\left(C_{n}, D_{n}\right)$ i.e. $\mathcal{P}=P \operatorname{Span}\left(C_{n}, D_{n}\right) Q$ for some pair $(P, Q) \in \mathrm{GL}_{n}(\mathbb{K}) \times \mathrm{GL}_{n+1}(\mathbb{K})$ :

Proposition 12. Let $A$ and $B$ in $M_{n, n+1}(\mathbb{K})$. Assume that every non-trivial linear combination of $A$ and $B$ over any field extension of $\mathbb{K}$ has rank $n$. Then there is a pair $(P, Q) \in G L_{n}(\mathbb{K}) \times G L_{n+1}(\mathbb{K})$ such that $A=P C_{n} Q^{-1}$ and $B=P D_{n} Q^{-1}$. 
Assuming this to be true, consider a basis $(A, B)$ of $\mathcal{P}$ and a pair $(P, Q)$ associated to it as in Proposition 12. Then a straightforward computation shows that $R \mathcal{H}_{2 n+1}(\mathbb{K}) R^{-1}=\mathcal{A}$ for $R=\left[\begin{array}{ll}P & 0 \\ 0 & Q\end{array}\right]$, which completes the proof of Theorem 5 .

Proof of Proposition 12. We let $x$ denote an indeterminate. We use the KroneckerWeierstrass reduction for pencils of matrices (see chapter XII of [2] and the appendix of [4] for the case of an arbitrary field). Since $A$ and $B$ have rank $n$ and belong to $\mathrm{M}_{n, n+1}(\mathbb{K})$, the canonical form of the pencil $A+x B$ cannot contain any block of the forms

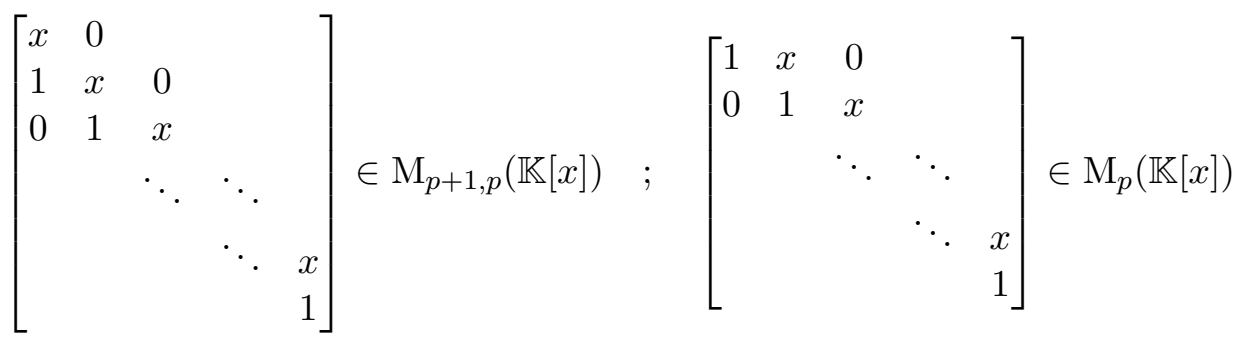

nor

$$
\left[\begin{array}{ccccc}
x & 1 & 0 & & \\
0 & x & 1 & & \\
& & \ddots & \ddots & \\
& & & \ddots & 1 \\
& & & & x
\end{array}\right] \in \mathrm{M}_{p}(\mathbb{K}[x])
$$

and contains at most one block of the form

$$
L_{p}:=\left[\begin{array}{ccccc}
1 & x & 0 & & \\
0 & 1 & x & & \\
& & \ddots & \ddots & \\
& & & 1 & x
\end{array}\right] \in \mathrm{M}_{p, p+1}(\mathbb{K}[x]) .
$$

It follows that there exists some $p \in \llbracket 0, n-1 \rrbracket$, some non-singular $C \in \mathrm{GL}_{p}(\mathbb{K})$ and some pair $(P, Q) \in \mathrm{GL}_{n}(\mathbb{K}) \times \mathrm{GL}_{n+1}(\mathbb{K})$ such that

$$
P^{-1}(A+x B) Q=\left[\begin{array}{cc}
C+x \cdot I_{p} & 0 \\
0 & L_{n-p}
\end{array}\right] .
$$


However, if $p>0$, then $\operatorname{rk}(A-\lambda B)<n$ for any eigenvalue $\lambda$ of $C$, which contradicts the assumptions. It follows that $p=0$, hence $P^{-1}(A+x B) Q=$ $L_{n}=C_{n}+x D_{n}$, which shows $A=P C_{n} Q^{-1}$ and $B=P D_{n} Q^{-1}$.

\section{Acknowledgements}

I would like to thank Saab Abou-Jaoudé for submitting me the original problem and Pierre Mazet for inspiring part of this work.

\section{References}

[1] L. Chambadal, J.L. Ovaert. Algèbre linaire et algèbre tensorielle. Dunod, 1968.

[2] F.A. Gantmacher. The Theory of Matrices, vol. 2. Chelsea Publishing Company, 1959.

[3] N. Jacobson, Basic Algebra II, 3rd edition. W.H. Freeman \& Company, 1980 .

[4] C. de Seguins Pazzis, Invariance of simultaneous similarity and equivalence of matrices under extension of the ground field, Linear Algebra Appl., 433-3 (2010), 618-624.

[5] D.A. Suprunenko, R.I. Tyshkevich. Commutative Matrices. Academic Press, 1968. 